EPJ Web of Conferences 47, 09005 (2013)

DOI: $10.1051 /$ epjconf/20134709005

(C) Owned by the authors, published by EDP Sciences, 2013

\title{
Pulsating stars harbouring planets
}

\author{
A. Moya ${ }^{\mathrm{a}}$ \\ Departamento de Astrofísica, Centro de Astrobiología (INTA-CSIC), PO Box 78, \\ 28691 Villanueva de la Cañada, Madrid, Spain
}

\begin{abstract}
Why bother with asteroseismology while studying exoplanets? There are several answers to this question. Asteroseismology and exoplanetary sciences have much in common and the synergy between the two opens up new aspects in both fields. These fields and stellar activity, when taken together, allow maximum extraction of information from exoplanet space missions. Asteroseismology of the host star has already proved its value in a number of exoplanet systems by its unprecedented precision in determining stellar parameters. In addition, asteroseismology allows the possibility of discovering new exoplanets through time delay studies. The study of the interaction between exoplanets and their host stars opens new windows on various physical processes. In this review I will summarize past and current research in exoplanet asteroseismology and explore some guidelines for the future.
\end{abstract}

\section{INTRODUCTION}

In a review devoted to the field of study of pulsating stars harbouring planets, S. Vauclair [40] wondered "why bother with asteroseismology in exoplanet research?". She gave several answers to this question. This review is based on further elaboration of these topics. In addition, I will discuss recent developments which relate to this question.

The potential of asteroseismology for the study of exoplanetary systems was already evident soon after the discovery of the first exoplanets. However, it is only in the last six years that the interaction between exoplanet research and asteroseismology began to show promising results. Let us begin by investigating some of the topics that resulted from Vauclair's question.

\section{SYNERGY: (ALMOST) THE SAME INSTRUMENTS ARE USED}

Asteroseismology and exoplanet detection are both based on the analysis of time series. Currently, the best way of detecting exoplanets is through radial velocity measurements (ground based observations) or the transit technique which demands a high photometric precision, mainly attained from space missions. Asteroseismology has the same requirements. Most of this review is devoted to the contribution of asteroseismology to exoplanet research. The contribution of exoplanet science to asteroseismology cannot be neglected since exoplanets may offer an explanation for some asteroseismological puzzles.

The synergy between the two fields will always co-exist in present and future exoplanet space missions. It is in the characterization of the host star that asteroseismology plays a key role [9, 17, 29].

\footnotetext{
ae-mail: amoya@cab.inta-csic.es
}

This is an Open Access article distributed under the terms of the Creative Commons Attribution License 2.0, which permits unrestricted use, distribution, and reproduction in any medium, provided the original work is properly cited. 


\section{NOISE: ONE PERSON'S NOISE IS ANOTHER PERSON'S SIGNAL}

Historically, the exoplanet community has treated the variability of the host star, whether activity or pulsation, as noise which difficults the detection of exoplanets [10]. Attempts have been made to reduce this "noise" using different techniques. However, sometimes the noise is a signal that can assist in the detection of the exoplanet and characterization of the central star. This is the idea behind the Plato Data Center, where light curves will be stored and studied by a collaboration between the exoplanet and asteroseismological communities.

\section{CHARACTERIZATION: OBTAIN PRECISE VALUES OF THE PARAMETERS OF EXOPLANETS-HOST STARS}

The study and characterization of stars hosting planets is an essential requirement in understanding the physics of exoplanets. This can be illustrated by a summary of the most successful techniques that are presently available for the detection of exoplanets. Currently, five main techniques are in use, but by far the most common are radial velocities, transits and direct imaging.

- Radial velocities. This technique uses the periodic variation in the radial velocity due to the gravitational perturbation of the orbiting planet. This method provides a lower limit on the mass ratio.

- Transits. An orbiting planet crossing the line of sight results in a shallow dip in the stellar light curve. Analysis of this eclipse gives the orbital parameters and ratio of the radii.

- Direct imaging. It is the direct detection of the planet in an image. The observational data is the planet luminosity. Measuring the luminosity of the planet and modelling the stellar age allows the mass of the planet to be determined.

It is clear that uncertainty in the general physical characteristics of the central star leads directly to uncertainty in the physical characteristics of the exoplanet [36]. There are a number of papers which discuss the use of asteroseismology in precise characterization of stellar parameters $[1,12,18,26,28$, 37, 38]. Most of these studies are for single main-sequence stars similar to the Sun.

All these studies stress the importance of asteroseismology. For solar-like stars the radius can be estimated with an uncertainty of about 3 percent, the mass to about 5 percent and the age to 5-10 percent. However, accuracy is not the same as precision; these uncertainties depend entirely on our understanding of stellar structure and evolution of the particular star.

Have we achieved the predicted precision in these parameters? A number of studies concerned with modeling of the observational data are available $[6,23]$. These studies show that the stellar radius is estimated to better than 1 percent for main-sequence solar-like stars. The mass is estimated with a precision of about 5 percent and the mean density to $<0.2$ percent. Currently, there are fifteen papers in which pulsating stars with planets are studied: [2, 3, 7, 8, 13-15, 19, 20, 24, 27, 31]. Most of these studies concern solar-like stars or post-MS stars with solar-like pulsations. Not all these papers discuss the pulsational characteristics of the host star in the same detail. Some highlights of these studies are itemized below.

- $\mu$ Arae. This was the first star where asteroseismology was applied to exoplanet research. The system consists of a giant exoplanet orbiting a solar-like star [3, 5, 33, 34].

- $\imath$ Horologii. This is another giant exoplanet orbiting a solar-like star [4, 20, 41]. The stellar age and He content estimated from asteroseismic analysis indicates membership of the Hyades cluster. This result led to a revision of the stellar mass and therefore to the mass of the planet.

- KIC 9904059. Asteroseismology of this star shows that a suspected planet is in fact a companion star [14]. This is an example of false positive found thanks to the asteroseismilogy [39].

- HAT-P-7 [7], HD 46375 [13], HD17156 [27]. These are good examples of accurate planet characterization using asteroseismology. 


\section{Hot Planets and Cool Stars}

- HR 8799. This is the only pulsating star with a planetary system that has been directly imaged [25]. The age of the system and the mass of the central star are the key parameters in this case.

It should be borne in mind that all these characterizations are very model dependent [21]. A better understanding of stellar physics is required to fully assess these predictions.

\section{PLANET DETECTION USING THE TIMING METHOD}

Asteroseismology can be also used to discover planets using the timing method [32] which requires an accurate astronomical clock. The general requirements for stellar pulsation modes to be used in this way are 1) very stable pulsational periods and amplitudes, 2) several independent high amplitude modes, and 3 ) the periods must be short. Some types of pulsating stars that may fulfill these requirements are $\mathrm{SdB}$ pulsators, roAp and $\delta$ Scuti stars. For example, a planet has been found in the SdB star V391 Peg by this method [31]. The EXOTIME international consortium is devoted to the use of the timing method for detecting planets around $\mathrm{SdB}$ stars $[22,30]$.

\section{INTERACTION BETWEEN STAR AND PLANET}

In addition, there are a number of on-going projects concerning star - planet interactions. For example, we know that most of the exoplanets so far discovered have metal-rich host stars, but why this is the case is not known [35]. We do not know whether the over-abundance of metals is intrinsic to the star or a result of accretion [3]. Stars with planets seem to have a lower Li abundance than stars without planets [16]. Asteroseismology may well shed light on these problems. An asteroseismic study of HR 8799 has shown, for the first time, a misalignment between the rotation axis of the host star and the orbital plane of the debris disk. In addition, there is misalignment with the orbital plane of the three known planets [42].

\section{FUTURE PROSPECTS}

The European Space Agency, in its "Cosmic Vision" plan, proposes four main objectives which are presented as questions. The first question is: what are the conditions for planet formation and the emergence of life? To answer this question, an advisory team suggested the following three steps: 1 ) discovering exoplanets, 2) characterization of these systems, and 3) the search for bio-markers [11]. As I have described in this review, asteroseismology is an important tool in planet detection and is essential for characterization of the system.

Future efforts will concentrate in the accurate characterization of host stars, especially for the brighter stars. Attempts should be made to discover more planetary systems around other types of pulsating stars and the use of asteroseismology to recognise false detections. The study of the interaction between star, planets and debris disk should be intensified. Finally, we need to apply asteroseismology to systems with exoplanets around very low mass stars and brown dwarfs.

\section{References}

[1] Basu S., Chaplin W. J., Elsworth Y., 2010, ApJ, 710, 1596

[2] Ballot J., et al., 2011, A\&A, 530, 97

[3] Bazot M., et al., 2005, A\&A, 440, 615

[4] Boisse I., et al., 2011, A\&A, 528, 4

[5] Bouchy F., et al., A\&A, 440, 609

[6] Chaplin W. J., et al., 2010, ApJ, 713, 169

[7] Christensen-Dalsgaard J., et al., 2010, ApJ, 713, 164

[8] Collier Cameron A., et al., 2010, MNRAS, 407, 507

[9] CoRoT, http://en.wikipedia.org/wiki/COROT 
[10] Dumusque X., et al., 2011, A\&A, 525, 140

[11] Exoplanet Roadmap Advisory Team (EPR-AT), http://sci.esa.int/eprat

[12] Gai N., et al., 2011, ApJ, 730, 63

[13] Gaulme P., et al., 2010, A\&A, 524, 47

[14] Gilliland R. L., et al., 2010, PASP, 122, 131

[15] Hatzes A. P., Zechmeister M., 2008, JPhCS, 118, 2016

[16] Israelian G., et al., 2009, Nature, 462, 189

[17] Kepler, http://kepler.nasa.gov/

[18] Kjeldsen H., Bedding T. R., Christensen-Dalsgaard J., 2009, IAU Symposium 253, 309

[19] Koen C., et al, 2003, MNRAS, 344, 1250

[20] Laymand M., Vauclair S., 2007, A\&A, 463, 657

[21] Lebreton Y., 2011, in "The 61st Fujihara seminar: Progress in solar/stellar physics with helio-and asteroseismology", (ed. H. Shibahashi), in press

[22] Lutz R., Schuh S., Silvotti R., 2011, AIPC, 1331, 155

[23] Metcalfe T. S., et al., 2010, ApJ, 723, 1583

[24] Moya A., et al., 2010, MNRAS, 406, 566

[25] Moya A., et al., 2010, MNRAS, 405, 81

[26] Mulet-Marquis C., et al., 2009, A\&A, 506, 153

[27] Nutzman P., et al., 2011, ApJ, 726, 3

[28] Quirion P. O., Christensen-Dalsgaard J., Arentoft T., 2010, ApJ, 725, 2176

[29] Plato, http://sci.esa.int/science-e/www/area/index.cfm?fareaid=104

[30] Schuh S., et al, 2010, Ap\&SS, 329, 231

[31] Silvotti R., et al., 2007, Nature, 449, 189

[32] Silvotti R., et al., 2011, AIPC, 1331, 133

[33] Soriano M., Vauclair S., 2008, JPhCS, 118, 2072

[34] Soriano M., Vauclair S., 2010, A\&A, 513, 49

[35] Sousa S. G., et al., 2011, A\&A, 533, 141

[36] Southworth J., 2011, MNRAS, 417, 2166

[37] Stello D., Kjeldsen H., Bendding T. R., 2007, ASPC, 366, 247S

[38] Stello D., et al., 2009, ApJ, 700, 1598

[39] Tingley B., Bonomo A. S., Deeg H. J., 2011, ApJ, 726, 112

[40] Vauclair S., 2008, Les Houches Winter School, arXiv:0809.0249

[41] Vauclair S., et al., 2008, A\&A, 482, 5

[42] Wright D., et al., 2011, ApJ, 278, 20 